\title{
Strong spatial dispersion in time-modulated dielectric media
}

\author{
Dani Torrent* \\ GROC, UJI, Institut de Noves Tecnologies de la Imatge (INIT), Universitat Jaume I, 12071 Castelló, Spain
}

(Received 29 September 2020; revised 19 November 2020; accepted 30 November 2020; published 8 December 2020)

\begin{abstract}
We present an effective medium description of time-modulated dielectric media. By taking the averaged fields over one modulation period, the relationship between them is derived, therefore defining the different constitutive parameters. In the most general situation, it is found that the effective material is described by means of a spatially and temporally dispersive transverse dielectric function and a constant longitudinal dielectric function. It has been also found that the frequency dependence in the former is weak, in comparison with its wavenumber dependence (spatial dispersion). Different physical consequences of this spatial dispersion are discussed, with special emphasis on the weak dispersion approximation and the limit in which it is found that the effective material behaves as a resonant and isotropic magnetodielectric medium with no additional longitudinal mode, as it is commonly found in spatially dispersive materials. Time-dependent media therefore opens an alternative way of designing dynamically tunable metamaterials.
\end{abstract}

DOI: 10.1103/PhysRevB.102.214202

\section{INTRODUCTION}

The study of naturally or artificially structured materials is a classic problem in physics and engineering. Also named composites, a countless number of theoretical and experimental methods have been developed to properly understand their properties [1]. In this context, metamaterials are a special type of composites, where the effective medium has extreme constitutive parameters mainly due to local resonances of the constitutive elements [2,3]. For electromagnetic materials, these extreme parameters imply the existence of simply or double negative materials, where a huge literature exist concerning their physics and applications [4-6]. However, the existence of strongly resonant effective properties is usually accompanied by more or less weakly spatially dispersive properties [7-11].

Metamaterials have recently evolved toward more complex structures, and the possibility of design of new materials based on the temporal modulation of the constitutive parameters has also been explored. Then it can be shown that, when a given medium presents time-modulated constitutive parameters, some effects like nonreciprocity, gain, and tunability can be easily achieved [12-18].

The concept of effective medium for a time-modulated material is similar to that of a spatially-modulated material $[19,20]$ in the sense that, when the modulation frequency (spatial or temporal) is fast and the operating wavelength and frequency cannot detect that modulation, we detect an effective medium with some averaged constitutive parameters. When the parameters are spatially modulated, we obtain an effective medium with a strong temporal dispersion (frequency dependence) but a weak spatial dispersion (wavenumber dependence).

\footnotetext{
*dtorrent@uji.es
}

In this work it will be shown that the temporal modulation exchanges these properties, and the effective medium has a strong spatial dispersion and a weak temporal dispersion. It will be shown that, after averaging the electromagnetic fields, the temporal modulation of the dielectric constant results in an effective material with a nonlocal transverse dielectric function, but a local longitudinal one. Analytical expressions will be derived for these two functions and some examples will be analyzed. Finally, it will be shown how, in the weak dispersion approximation, the effective material behaves as an isotropic magnetodielectric medium, a property achieved so far mainly by complex three-dimensional metamaterials [21-24]. Therefore, the temporal modulation of the dielectric constant is an excellent alternative for the realization of complex materials with extreme electromagnetic properties.

The paper is organized as follows: Section II presents the homogenization method and the expressions for the nonlocal dielectric function. Section III analyzes the spatially dispersive dielectric function and some of its properties. In Sec. IV the consequences of the spatial dispersion for finite slabs in both space and time are discussed. In Sec. V the artificial magnetic effect due to the weak dispersion approximation is analyzed. Finally, Sec. VI summarizes the work.

\section{NONLOCAL DIELECTRIC FUNCTION}

The evolution of the electromagnetic field in matter excited by an external current $\boldsymbol{J}_{\text {ext }}$ and charge density $\rho_{\text {ext }}$ is described by means of Maxwell's equations,

$$
\begin{gathered}
\nabla \times \boldsymbol{E}=-\partial_{t} \boldsymbol{B}, \\
\nabla \times \boldsymbol{H}=\partial_{t} \boldsymbol{D}+\boldsymbol{J}_{\mathrm{ext}}, \\
\nabla \cdot \boldsymbol{D}=\rho_{\mathrm{ext}}, \\
\nabla \cdot \boldsymbol{B}=0,
\end{gathered}
$$


whose solution can be obtained only once we know the constitutive equations relating the fields $\boldsymbol{H}, \boldsymbol{B}, \boldsymbol{E}$ and $\boldsymbol{D}$. In the media we aim to study, these constitutive equations are the corresponding ones to a nonmagnetic material with a timedependent electrical permittivity $\varepsilon(t)$, then we will have that $\boldsymbol{E}(\boldsymbol{r}, t)=\varepsilon^{-1}(t) \boldsymbol{D}(\boldsymbol{r}, t)$ and $\boldsymbol{B}(\boldsymbol{r}, t)=\mu_{0} \boldsymbol{H}(\boldsymbol{r}, t)$.

We will assume as well that the function $\epsilon(t)$ is $T$ periodic in time, and that the modulation frequency $v_{M}=1 / T$ is larger than the operating frequency $\omega$ of the external currents and charges. We can assume then that the response of the system will be a smooth function modulated by a fast function whose average in a period $T$ will be zero. The relationship between these averaged fields will define the effective constitutive parameters of the material, identically as it happens in spatially periodic media.

Let us then assume that the time-dependent dielectric function $\varepsilon(t)$ and its inverse $\varepsilon^{-1}(t)$ can be expanded in a Fourier series of the form

$$
f(t)=\sum_{n} f_{n} e^{-2 i \pi n t / T},
$$

where $f_{n}$ are labeled $\varepsilon_{n}$ and $\varepsilon_{n}^{-1}$ for $\varepsilon(t)$ and $\varepsilon^{-1}(t)$, respectively. Notice that with this notation for $n=0$ we have that $\varepsilon_{0}=\langle\varepsilon(t)\rangle$ and $\varepsilon_{0}^{-1}=\left\langle\varepsilon(t)^{-1}\right\rangle$, not to be confused with the permittivity of vacuum.

The external current and density are the ones selecting the operating frequency $\omega$ and wavenumber $\boldsymbol{k}$, thus we assume

$$
\begin{aligned}
& \boldsymbol{J}_{\mathrm{ext}}=\boldsymbol{J}_{0} e^{i \boldsymbol{k} \cdot \boldsymbol{r}} e^{-i \omega t}, \\
& \rho_{\mathrm{ext}}=\rho_{0} e^{i \boldsymbol{k} \cdot \boldsymbol{r}} e^{-i \omega t},
\end{aligned}
$$

and we know that in this case the solution for the fields will be of the form

$$
\begin{aligned}
\boldsymbol{u}(\boldsymbol{r}, t) & =e^{i \boldsymbol{k} \cdot \boldsymbol{r}} e^{-i \omega t} \sum_{n} \boldsymbol{u}_{n} e^{-2 i \pi n t / T} \\
& =e^{i \boldsymbol{k} \cdot \boldsymbol{r}} e^{-i \omega t} \boldsymbol{u}_{0}+e^{i \boldsymbol{k} \cdot \boldsymbol{r}} e^{-i \omega t} \sum_{n \neq 0} \boldsymbol{u}_{n} e^{-2 i \pi n t / T},
\end{aligned}
$$

where $\boldsymbol{u}=\boldsymbol{E}, \boldsymbol{D}, \boldsymbol{B}$ and $\boldsymbol{H}$. Therefore, the response of the electromagnetic field to an external field of wavenumber $\boldsymbol{k}$ and frequency $\omega$ is composed of a slow component $\boldsymbol{u}_{0}$ and a fast modulation $\boldsymbol{u}_{n}$ for $n \neq 0$. For a fast modulation frequency $v_{M}=1 / T$ we can interpret the $n=0$ as the averaged "observable" terms, so that their evolution will define the evolution of the effective material.

Then the relationship between the $n=0$ terms in the above expansions is

$$
\begin{gathered}
\boldsymbol{k} \times \boldsymbol{E}_{0}=\omega \boldsymbol{B}_{0}, \\
\boldsymbol{k} \times \boldsymbol{H}_{0}=-\omega \boldsymbol{D}_{0}-i \boldsymbol{J}_{0}, \\
i \boldsymbol{k} \cdot \boldsymbol{D}_{0}=\rho_{0}, \\
\boldsymbol{k} \cdot \boldsymbol{B}_{0}=0 .
\end{gathered}
$$

These expressions show that the $n=0$ component of the field expansion satisfies Maxwell's equation in Fourier space, as expected. The objective now is to find the relationship between these components, which will define the effective constitutive parameters of the medium. The effective magnetic permeability can be trivially found, since

$$
\boldsymbol{B}_{0}=\mu_{0} \boldsymbol{H}_{0},
$$

although it will be seen later that, due to the spatial dispersion in the effective dielectric constant, an effective magnetic permeability will be found. Concerning the relationship between $\boldsymbol{E}_{0}$ and $\boldsymbol{D}_{0}$, we see that this can be written as

$$
\boldsymbol{E}_{0}=\varepsilon(t)^{-1} \boldsymbol{D}_{0}=\varepsilon_{0}^{-1} \boldsymbol{D}_{0}+\sum_{n \neq 0} \varepsilon_{-n}^{-1} \boldsymbol{D}_{n} .
$$

Thus, we need the relationship between $\boldsymbol{D}_{n}$ and $\boldsymbol{D}_{0}$ in order to properly define an effective constitutive equation. This relationship is found from Maxwell's equations, since the wave equation for $\boldsymbol{D}$ is

$$
\varepsilon^{-1}(t) \nabla \times \nabla \times \boldsymbol{D}=-\mu_{0} \partial_{t t}^{2} \boldsymbol{D}-\mu_{0} \partial_{t} \boldsymbol{J}_{\mathrm{ext}},
$$

which, after using Eqs. (6) and (8), is equivalent to

$$
-\sum_{m} \varepsilon_{n-m}^{-1} \boldsymbol{k} \times \boldsymbol{k} \times \boldsymbol{D}_{m}=\mu_{0} \Omega_{n}^{2} \boldsymbol{D}_{n}+i \mu_{0} \omega \boldsymbol{J}_{0} \delta_{n 0},
$$

where we have defined the displaced frequency $\Omega_{n}=\omega+$ $\frac{2 n \pi}{T}$. For $n=0$ the above equation is

$$
-\varepsilon_{0}^{-1} \boldsymbol{k} \times \boldsymbol{k} \times \boldsymbol{D}_{0}+\sum_{m \neq 0} \varepsilon_{-m}^{-1} k^{2} \boldsymbol{D}_{m}=\mu_{0} \omega^{2} \boldsymbol{D}_{0}+i \mu_{0} \omega \boldsymbol{J}_{0},
$$

since we have from Eq. (3) that $i \boldsymbol{k} \cdot \boldsymbol{D}_{n}=\delta_{n 0} \rho_{0}$. Similarly, for $n \neq 0$ we have

$$
-\varepsilon_{n}^{-1} \boldsymbol{k} \times \boldsymbol{k} \times \boldsymbol{D}_{0}+\sum_{m \neq 0} \varepsilon_{n-m}^{-1} k^{2} \boldsymbol{D}_{m}=\mu_{0} \Omega_{n}^{2} \boldsymbol{D}_{n},
$$

from which we obtain the desired relationship between $\boldsymbol{D}_{m}$ and $\boldsymbol{D}_{0}$,

$$
\boldsymbol{D}_{m}=-\sum_{n \neq 0} \Gamma_{m n}^{D} \varepsilon_{n}^{-1} \boldsymbol{k} \times \boldsymbol{k} \times \boldsymbol{D}_{0},
$$

with

$$
\Gamma_{m n}^{D}=\left(\mu_{0} \Omega_{n}^{2} \delta_{m n}-\varepsilon_{n-m}^{-1} k^{2}\right)^{-1} .
$$

We therefore have obtained the fundamental relationship between the fast terms and the average field $\boldsymbol{D}_{0}$, thus Eq. (19) can now be introduced into Eq. (14) and we get, after some algebra,

$$
\boldsymbol{E}_{0}=\varepsilon_{T}^{-1}(\omega, \boldsymbol{k}) \boldsymbol{D}_{0}+\left(\varepsilon_{L}^{-1}-\varepsilon_{T}^{-1}(\omega, \boldsymbol{k})\right) \boldsymbol{u}_{\boldsymbol{k}} \boldsymbol{D}_{0} \cdot \boldsymbol{u}_{\boldsymbol{k}},
$$

where we have defined the transverse and longitudinal inverse dielectric constants $\varepsilon_{T}^{-1}$ and $\varepsilon_{L}^{-1}$, respectively, as

$$
\begin{gathered}
\varepsilon_{T}^{-1}(\omega, \boldsymbol{k})=\left\langle\varepsilon^{-1}\right\rangle+k^{2} \sum_{n, m \neq 0} \varepsilon_{-m}^{-1} \Gamma_{m n}^{D} \varepsilon_{n}^{-1}, \\
\varepsilon_{L}^{-1}=\left\langle\varepsilon^{-1}\right\rangle,
\end{gathered}
$$

which clearly satisfies

$$
\begin{gathered}
\boldsymbol{k} \times \boldsymbol{E}_{0}=\varepsilon_{T}^{-1}(\omega, \boldsymbol{k}) \boldsymbol{k} \times \boldsymbol{D}_{0}, \\
\boldsymbol{k} \cdot \boldsymbol{E}_{0}=\varepsilon_{L}^{-1} \boldsymbol{k} \cdot \boldsymbol{D}_{0} .
\end{gathered}
$$


We therefore see that the effective material behaves as a material with a nonlocal transverse dielectric response but a local longitudinal response. The longitudinal response is independent of both the frequency and the wavenumber, while the transverse dielectric function depends on both of them, i.e., it is nonlocal in space and time. However, as can be seen from Eq. (20), this dependence is of the form $\omega+2 n \pi / T$ for $n \neq 0$, which actually implies that, if the modulation frequency $v_{M}=1 / T$ of the medium is larger than the operating frequency, this dependence will disappear and we will have nonlocality in space only. This is the opposite situation as is typically found in classical periodic materials, where nonlocality in time (frequency-dependent dielectric constant) is stronger than nonlocality in space, for identical reasons as those considered here, except for layered or wire media, as will be discussed later.

We can check the consistency of the definition of $\varepsilon_{T}^{-1}$ by inserting Eq. (19) into Eq. (17), since we obtain

$$
-\varepsilon_{T}^{-1}(\omega, \boldsymbol{k}) \boldsymbol{k} \times \boldsymbol{k} \times \boldsymbol{D}_{0}=\mu_{0} \omega^{2} \boldsymbol{D}_{0}+i \mu_{0} \omega \boldsymbol{J}_{0},
$$

which is identical to the wave equation we would obtain from Eqs. (9) and (10) using the constitutive equation derived in Eq. (21).

We can proceed in a similar way to obtain an alternative expression for $\varepsilon_{T}(\omega, \boldsymbol{k})$. Thus, the wave equation for the $\boldsymbol{H}$ field is given by

$$
\nabla \times \nabla \times \boldsymbol{H}=-\mu_{0} \partial_{t}\left(\epsilon(t) \partial_{t} \boldsymbol{H}\right)+\nabla \times \boldsymbol{J}_{\text {ext }},
$$

again using Eqs. (6) and (8) it becomes

$$
k^{2} \boldsymbol{H}_{n}=\mu_{0} \sum_{m} \Omega_{n} \varepsilon_{n-m} \Omega_{m} \boldsymbol{H}_{m}+i \boldsymbol{k} \times \boldsymbol{J}_{0} \delta_{n 0} .
$$

As before, we can split these equations into the $n=0$ component,

$$
k^{2} \boldsymbol{H}_{0}=\mu_{0}\langle\varepsilon\rangle \omega^{2} \boldsymbol{H}_{0}+\mu_{0} \omega \sum_{m \neq 0} \varepsilon_{-m} \Omega_{m} \boldsymbol{H}_{m}+i \boldsymbol{k} \times \boldsymbol{J}_{0},
$$

and the $n \neq 0$ set of equations,

$$
k^{2} \boldsymbol{H}_{n}=\mu_{0} \omega \varepsilon_{n} \Omega_{n} \boldsymbol{H}_{0}+\mu_{0} \sum_{m \neq 0} \Omega_{n} \varepsilon_{n-m} \Omega_{m} \boldsymbol{H}_{m},
$$

from which we can obtain the expression analog of Eq. (19), but for $\boldsymbol{H}_{m}$,

$$
\boldsymbol{H}_{m}=\mu_{0} \omega \sum_{n \neq 0} \Gamma_{m n}^{H} \Omega_{n} \varepsilon_{n} \boldsymbol{H}_{0},
$$

where now we have defined

$$
\Gamma_{m n}^{H}=\left(k^{2} \delta_{m n}-\mu_{0} \Omega_{n} \varepsilon_{n-m} \Omega_{m}\right)^{-1} .
$$

Equation (29) is now

$$
k^{2} \boldsymbol{H}_{0}=\mu_{0} \varepsilon_{T}(\omega, \boldsymbol{k}) \omega^{2} \boldsymbol{H}_{0}+i \boldsymbol{k} \times \boldsymbol{J}_{0},
$$

where

$$
\varepsilon_{T}(\omega, \boldsymbol{k})=\langle\varepsilon\rangle+\mu_{0} \sum_{m, n \neq 0} \Omega_{n} \varepsilon_{n} \Gamma_{n m}^{H} \varepsilon_{-m} \Omega_{m} .
$$

We therefore arrive at two possible definitions of $\varepsilon_{T}(\omega, \boldsymbol{k})$, as given from Eqs. (22) and (34), and we would like to check if these are equivalent or not. To do so we should prove that
$\varepsilon_{T}(\omega, \boldsymbol{k}) \varepsilon_{T}(\omega, \boldsymbol{k})^{-1}=1$. Then, if we use the time-dependent constitutive equation and introduce it in Eq. (2), we get

$$
\nabla \times \boldsymbol{D}=-\mu_{0} \varepsilon(t) \partial_{t} \boldsymbol{H},
$$

which in $\omega-\boldsymbol{k}$ space is

$\boldsymbol{k} \times \boldsymbol{D}_{0}=\omega \mu_{0} \varepsilon_{0} \boldsymbol{H}_{0}+\mu_{0} \sum_{m \neq 0} \Omega_{m} \varepsilon_{-m} \boldsymbol{H}_{m}=\omega \mu_{0} \varepsilon_{T}(\omega, \boldsymbol{k}) \boldsymbol{H}_{0}$.

However, from Eqs. (24) and (9) we get

$$
\varepsilon_{T}^{-1}(\omega, \boldsymbol{k}) \boldsymbol{k} \times \boldsymbol{D}_{0}=\omega \mu_{0} \boldsymbol{H}_{0},
$$

which actually implies that

$$
\varepsilon_{T}^{-1}(\omega, \boldsymbol{k}) \varepsilon_{T}(\omega, \boldsymbol{k})=1,
$$

as expected.

We can now use the expressions of Eqs. (22) or (34) for our convenience, as will be shown below. For instance, if we want to determine the limit of low $\omega$ and $\boldsymbol{k}$, what we could call the "static limit," it is more suitable to use Eq. (22), so that we obtain trivially

$$
\varepsilon_{T}^{-1}=\left\langle\varepsilon^{-1}\right\rangle=\varepsilon_{L}^{-1} .
$$

The material is then a homogeneous dielectric material with an averaged reciprocal dielectric constant, with identical transverse and longitudinal responses. The above result generalizes the result obtained in reference [19] for a layered time-dependent material, showing the consistency of this approach.

\section{FREQUENCY-INDEPENDENT SPATIALLY DISPERSIVE DIELECTRIC FUNCTION}

Let us consider now the case of a weak modulation changing the dielectric constant from $\langle\varepsilon\rangle+\Delta$ to $\langle\varepsilon\rangle-\Delta$ harmonically at a frequency $v_{M}=1 / T$. We therefore have

$$
\varepsilon(t)=\langle\varepsilon\rangle+\Delta \cos \left(2 \pi v_{M} t\right) .
$$

We can assume first that $2 \pi v_{M} \gg \omega$ to focus the analysis on the spatially dispersive properties of the material. Since all the Fourier components for $n \neq 0, \pm 1$ are zero, we can restrict our analysis to these orders. It is clear now that the $\Gamma^{H}$ matrix is diagonal with elements

$$
\Gamma_{ \pm 1 \pm 1}^{H}=\frac{1}{k^{2}-\mu_{0}\langle\varepsilon\rangle 4 \pi^{2} v_{M}^{2}},
$$

therefore the effective dielectric constant is given by

$$
\varepsilon_{T}(\boldsymbol{k})=\langle\varepsilon\rangle+\frac{\Delta^{2}}{2\langle\varepsilon\rangle} \frac{k_{M}^{2}}{k^{2}-k_{M}^{2}},
$$

where $k_{M}^{2}=4 \pi^{2} v_{M}^{2} \mu_{0}\langle\varepsilon\rangle$.

The above dielectric constant is similar to that found for the so-called "wire media", in which propagation takes place parallel to a periodic distribution of cylinders [25-27]. This similarity has a clear explanation: in any periodic medium, we will have factors of the form $k+2 \pi / a$ in the homogeneization process, so that the dependence on $k$ will be, in general, small. However, since in wire media the periodicity along the $z$ axis is "broken" by letting $a \rightarrow \infty$, we have a strong dependence on $k$. Similarly, the time periodicity of 
(a)

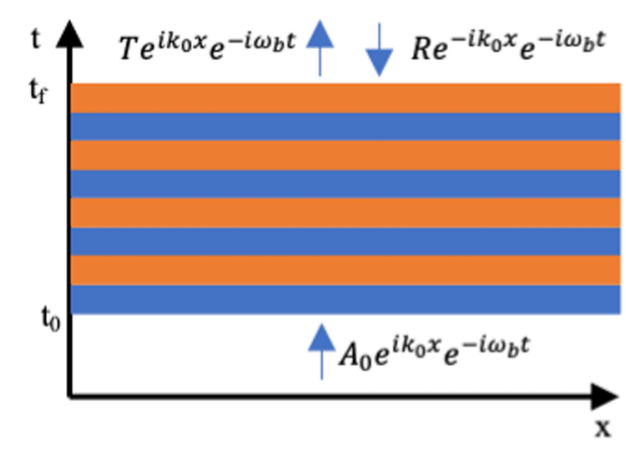

(b)

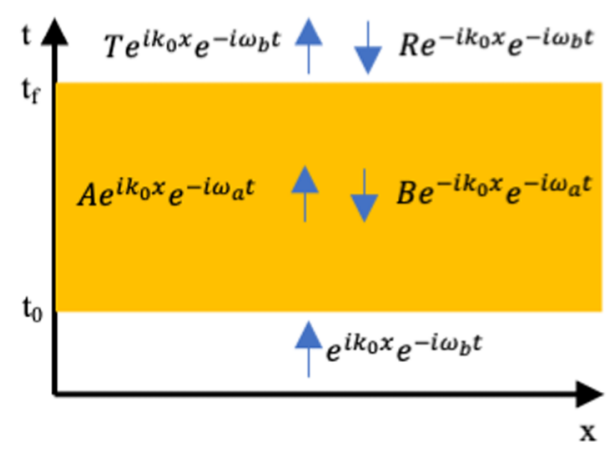

(c)

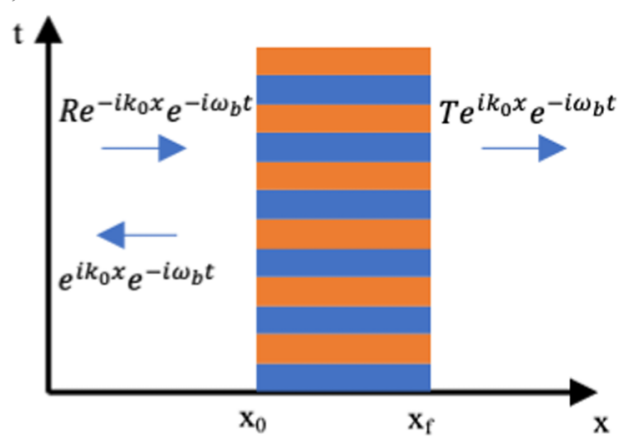

(d)

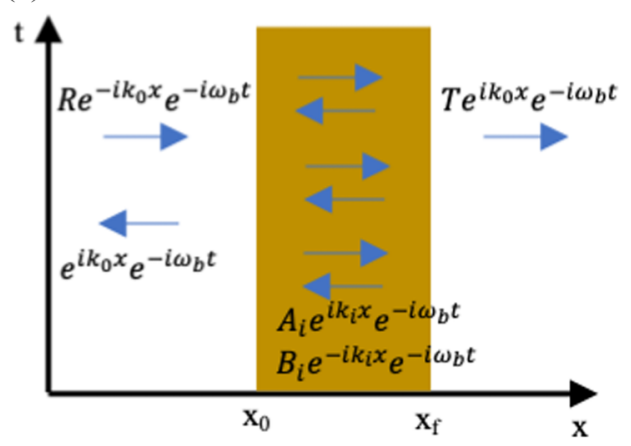

FIG. 1. Space-time representation of different modulation scenarios. (a) Homogeneous material modulated in time during a period $t=$ $t_{f}-t_{0}$. (b) Homogenization of the periodic modulation presented in panel $a$. (c) Periodic modulation in time of a finite slab placed between $x=x_{0}$ and $x=x_{f}$. (d) Homogenization of the structure presented in panel $d$.

the material is generally "broken", for that reason we have a strong dependence on $\omega$ in composites. However, in this case the "broken" periodicity is along the full space, while we still have periodicity along the time axis, thus we find a strong $k$ dependence and, consequently, strong nonlocality.

The functional form of Eq. (42) is not unique of the weak harmonic modulation, as will be demonstrated below. The matrix $\Gamma^{H}$ is defined in Eq. (32) as the inverse of the matrix $M$ given by

$$
M_{n m}=k^{2} \delta_{m n}-\mu_{0} \Omega_{n} \varepsilon_{n-m} \Omega_{m} .
$$

Since this matrix is Hermitian, we can use the eigendecomposition of a matrix and express the inverse as a function of the eigenvectors $\boldsymbol{v}_{\ell}$ and eigenvalues $\lambda_{\ell}$ of $\boldsymbol{M}$. Since matrix $\boldsymbol{M}$ has the form $\boldsymbol{M}=\boldsymbol{I} k^{2}-\chi$, its eigenvalues are $\lambda=k^{2}-\lambda^{\prime}$, with $\lambda^{\prime}$ being the $k$-independent eigenvalues of $\chi$, thus we have that

$$
\Gamma^{H}=\sum_{\ell} \frac{\boldsymbol{v}_{\ell} \otimes \boldsymbol{v}_{\ell}^{\dagger}}{k^{2}-\lambda_{\ell}^{\prime}}
$$

and Eq. (34) is

$$
\varepsilon_{T}(\boldsymbol{k})=\langle\varepsilon\rangle+\mu_{0} \sum_{\ell} \sum_{m, n \neq 0} \Omega_{n} \varepsilon_{n} \frac{\boldsymbol{v}_{\ell} \otimes \boldsymbol{v}_{\ell}^{\dagger}}{k^{2}-\lambda_{\ell}^{\prime}} \varepsilon_{-m} \Omega_{m} .
$$

In the denominator of the above expression the $\lambda_{\ell}^{\prime}$ are independent of $k$, and it can be easily shown that the numerator is as well independent of $k$, since

$$
\frac{\partial \boldsymbol{v}_{\ell}}{\partial k}=\left(\lambda_{\ell} \boldsymbol{I}-\boldsymbol{M}\right)^{\dagger} \frac{\partial \boldsymbol{M}}{\partial k} \boldsymbol{v}_{\ell}=0 .
$$

Then, Eq. (45) shows that the general form of $\varepsilon(\boldsymbol{k})$ is similar to the weak harmonic modulation but with more poles. In the above expressions we have ignored the possible dependence on frequency of $\varepsilon$, however we have previously discussed that this dependence is weak, but if it has to be included it will appear through the eigenvalues and eigenvectors of the expansion.

\section{SPACE-TIME REPRESENTATION OF FINITE MATERIALS}

It is worthwhile now to discuss some physics concerning the time modulation of the dielectric constant, which will help us to understand the possible implications of Eq. (42). Let us consider the situation illustrated in Fig. 1(a). What we see is a spatially homogeneous material with some dielectric constant $\varepsilon_{b}$ and, at $t=t_{0}$, a periodic modulation is applied until $t=t_{f}$. This situation is similar to that analyzed for acoustic waves in $[14,28]$. We can assume that we are far away from the band gap, where the material would be unstable, and that the conditions for the application of the effective medium condition hold. Then we are in the situation described in Fig. 1(b). We can see how, for $t<t_{0}$, a wave is propagating through the material with some frequency $\omega_{b}$ and wavenumber $k_{b}$. Once the modulation begins, we excite a "transmitted" and "reflected" wave, but the wavenumber of these waves continues being $k_{b}$, and it is the frequency that the quantity has changed $[14,19]$. To obtain the new frequency in the effective material we need to solve the dispersion relation

$$
\omega^{2}=\varepsilon_{T}^{-1}(\boldsymbol{k}) k^{2},
$$


where we have assumed that there is no dependence of $\varepsilon$ on $\omega$. Thus, the time slab has a similar behavior than the spatial slab, since once the modulation stops we will have transmitted and reflected waves whose relative amplitude can present gain or loss, as explained in [14], but spatial dispersion does not change the physics of the problem. The only thing that changes is its resonant-like nature, similarly as for spatially modulated materials.

However, the common situation is to have a spatially limited material (slab), even if we have an additional modulation in time. Let us therefore consider the situation shown in Fig. 1(c), where we see a periodically modulated material in time, but limited to the region $x \in\left[x_{0}, x_{f}\right]$. The full wave analysis of this situation is rather complex, as can be seen for the geometry of the domains involved, however it is now where the effective medium concept is especially useful, as it is shown in Fig. 1(d). The problem now is limited to a classical transmission-reflection problem, but now the operating frequency $\omega_{b}$ is indeed a conserved quantity, since any time dependence of the geometry has been averaged. An additional difficulty appears in this case, since spatial dispersion usually involves additional propagating modes that requires the use of additional boundary conditions, as studied in many works [29-32], although a recent approach based on an elastodynamic model for spatially dispersive materials could be more adequate for isotropic strongly dispersive materials with a transverse dielectric response [33].

\section{ISOTROPIC ARTIFICIAL MAGNETISM}

The only treatable situation in which additional boundary conditions are not required is the so called "weak" dispersion approximation, which assumes that $k$ is a small quantity so that we can expand $\varepsilon_{T}(\boldsymbol{k})$ as

$$
\varepsilon_{T}(\boldsymbol{k}) \approx \varepsilon_{T}(0)+\gamma k^{2},
$$

since in our case it is clear that there is no linear term in $k$. Then, according to our response model, we have

$$
\boldsymbol{D}_{0}=\varepsilon_{T}(\boldsymbol{k})+\left(\varepsilon_{L}-\varepsilon_{T}(\boldsymbol{k})\right) \boldsymbol{u}_{\boldsymbol{k}} \boldsymbol{D}_{0} \cdot \boldsymbol{u}_{\boldsymbol{k}} .
$$

We showed before that for $\boldsymbol{k}=\mathbf{0}$ and $\omega=0$ we have $\varepsilon_{T}=\varepsilon_{L}$, so that the above expression is approximated to

$$
\boldsymbol{D}_{0} \approx \varepsilon_{T}(\mathbf{0}) \boldsymbol{E}_{0}-\gamma \boldsymbol{k} \times \boldsymbol{k} \times \boldsymbol{E}=\varepsilon_{T}(\mathbf{0}) \boldsymbol{E}_{0}-\omega \gamma \boldsymbol{k} \times \boldsymbol{B}_{0},
$$

which gives an "artificial" isotropic magnetic response $\mu(\omega)$

$$
\mu(\omega)=\frac{\mu_{0}}{1-\mu_{0} \gamma \omega^{2}} .
$$

The parameter $\gamma$ then has a clear physical interpretation: its presence induces a resonant magnetic response of frequency $\omega_{R}$ given by

$$
\omega_{R}=\frac{1}{\mu_{0} \gamma} .
$$

If we want our model to be consistent near the resonant regime, we require that $\omega_{R}<<\omega_{M}$, otherwise we should include temporal dispersion in $\epsilon_{T}$ to obtain a more accurate description. This is the case in resonant metamaterials as well, where the frequency dependence of the effective $\epsilon$ is not always enough to fully describe the material near resonances.

It is worth mentioning that isotropic artificial magnetism has been a topic of intense research in the domain of metamaterials, especially at optical frequencies, and complex three-dimensional structures are in general required to achieve this interesting property [21-24]. The temporal modulation of the dielectric constant is therefore an interesting alternative, although it presents different and obvious technical difficulties.

Expanding Eq. (42), it is easy to see that, for a weak periodic modulation,

$$
\mu(\omega)=\frac{\mu_{0}}{1-\frac{\Delta^{2}}{\langle\varepsilon\rangle^{2}} \frac{\omega^{2}}{\omega_{M}^{2}}} .
$$

Finally, Eq. (22) allows us to obtain a very nice expression of the Taylor expansion of $\varepsilon_{T}^{-1}(\boldsymbol{k})$ in the general case, since it is easy to show

$$
\varepsilon_{T}^{-1}(\boldsymbol{k}) \approx\left\langle\varepsilon^{-1}\right\rangle+k^{2} \sum_{n \neq 0} \frac{\left|\varepsilon_{n}^{-1}\right|^{2}}{\mu_{0} \Omega_{n}^{2}},
$$

from which we obtain the expression for $\gamma$ in the most general case,

$$
\gamma=\frac{1}{\left\langle\varepsilon^{-1}\right\rangle^{2}} \sum_{n \neq 0} \frac{\left|\varepsilon_{n}^{-1}\right|^{2}}{\mu_{0} \Omega_{n}^{2}} .
$$

\section{SUMMARY}

In summary, we have derived an effective medium theory for time-modulated dielectric materials. It has been found that, in general, the fields can be decomposed into averaged and fast-modulated components, and the relationship between the slow components of the fields define the effective parameters of the material. It then has been demonstrated that the effective dielectric constant has a transverse component presenting a strong spatial dispersion but a weak temporal one, contrarily as space-modulated metamaterials, where the dominant effect is temporal dispersion. Analytical expressions have been derived for several examples, and the consequences of this strong spatial dispersion have been discussed under different scenarios, with especial emphasis in the so-called "weak dispersion approximation," in which an artificial isotropic magnetic response has been found. Since the modulation frequency is, in principle, a dynamic quantity easier to control in real time than the spatial modulation, we consider that this approach opens the door to a new class of dynamically tunable metamaterials.

\section{ACKNOWLEDGMENTS}

Dani Torrent acknowledges financial support through the "Ramón y Cajal" fellowship under Grant No. RYC-201621188 and to the Ministry of Science, Innovation and Universities through Project No. RTI2018- 093921-A-C42. 
[1] G. W. Milton and A. Sawicki, Appl. Mech. Rev. 56, B27 (2003).

[2] C. R. Simovski and S. A. Tretyakov, Phys. Rev. B 75, 195111 (2007).

[3] A. Alu, Phys. Rev. B 84, 075153 (2011).

[4] T. J. Cui, D. R. Smith, and R. Liu, Metamaterials (Springer, Heidelberg, 2010).

[5] W. Cai and V. M. Shalaev, Optical Metamaterials, Vol. 10 (Springer, Heidelberg, 2010).

[6] R. Marqués, F. Martin, and M. Sorolla, Metamaterials with Negative Parameters: Theory, Design, and Microwave Applications, Vol. 183 (John Wiley \& Sons, Hoboken, 2011).

[7] A. I. Căbuz, D. Felbacq, and D. Cassagne, Phys. Rev. A 77, 013807 (2008).

[8] S. S. Kruk, D. A. Powell, A. Minovich, D. N. Neshev, and Y. S. Kivshar, Opt. Express 20, 15100 (2012).

[9] A. D. Yaghjian, A. Alù, and M. G. Silveirinha, Photonics and Nanostructures-Fundamentals and Applications 11, 374 (2013).

[10] R.-L. Chern, Opt. Express 21, 16514 (2013).

[11] D. Torrent, Y. Pennec, and B. Djafari-Rouhani, Phys. Rev. B 92 , 174110 (2015).

[12] A. G. Hayrapetyan, J. B. Götte, K. K. Grigoryan, S. Fritzsche, and R. G. Petrosyan, J. Quant. Spectrosc. Radiat. Transfer 178, 158 (2016).

[13] H. Nassar, H. Chen, A. Norris, and G. Huang, Extreme Mech. Lett. 15, 97 (2017).

[14] D. Torrent, W. J. Parnell, and A. N. Norris, Phys. Rev. B 97, 014105 (2018).

[15] D. Torrent, O. Poncelet, and J.-C. Batsale, Phys. Rev. Lett. 120, 125501 (2018).

[16] Y. Chen, X. Li, H. Nassar, A. N. Norris, C. Daraio, and G. Huang, Phys. Rev. Appl. 11, 064052 (2019).

[17] G. Trainiti, Y. Xia, J. Marconi, G. Cazzulani, A. Erturk, and M. Ruzzene, Phys. Rev. Lett. 122, 124301 (2019).
[18] C. Croënne, J. Vasseur, O. Bou Matar, A.-C. Hladky-Hennion, and B. Dubus, J. Appl. Phys. 126, 145108 (2019).

[19] V. Pacheco-Peña and N. Engheta, Nanophotonics 9, 379 (2020).

[20] P. A. Huidobro, M. G. Silveirinha, E. Galiffi, and J. Pendry, arXiv:2009.10479.

[21] C. R. Simovski and S. A. Tretyakov, Phys. Rev. B 79, 045111 (2009).

[22] S. Mühlig, A. Cunningham, S. Scheeler, C. Pacholski, T. Bürgi, C. Rockstuhl, and F. Lederer, ACS nano 5, 6586 (2011).

[23] V. Ponsinet, P. Barois, S. M. Gali, P. Richetti, J.-B. Salmon, A. Vallecchi, M. Albani, A. Le Beulze, S. Gomez-Grana, E. Duguet, S. Mornet, and M. Treguer-Delapierre, Phys. Rev. B 92, 220414(R) (2015).

[24] S. Gómez-Graña et al., Materials Horizons 3, 596 (2016).

[25] S. Maslovski, S. Tretyakov, and P. Belov, Microwave and Optical Technology Letters 35, 47 (2002).

[26] P. A. Belov, R. Marques, S. I. Maslovski, I. S. Nefedov, M. Silveirinha, C. R. Simovski, and S. A. Tretyakov, Phys. Rev. B 67, 113103 (2003).

[27] S. I. Maslovski and M. G. Silveirinha, Phys. Rev. B 80, 245101 (2009).

[28] A. Hayrapetyan, K. Grigoryan, R. Petrosyan, and S. Fritzsche, Ann. Phys. 333, 47 (2013).

[29] C.-S. Ting, M. Frankel, and J. Birman, Solid State Commun. 17, 1285 (1975).

[30] P. Halevi and G. Hernández-Cocoletzi, Phys. Rev. Lett. 48, 1500 (1982).

[31] M. G. Silveirinha, New J. Phys. 11, 113016 (2009).

[32] S. I. Maslovski, T. A. Morgado, M. G. Silveirinha, C. S. Kaipa, and A. B. Yakovlev, New J. Phys. 12, 113047 (2010).

[33] J. V. Alvarez, B. Djafari-Rouhani, and D. Torrent, Phys. Rev. B 102, 115308 (2020). 